\title{
Some Classes of Function Spaces, Their Properties, and Their Applications 2014
}

\author{
Józef Banaś, ${ }^{1}$ Janusz Matkowski, ${ }^{2}$ Nelson Merentes, ${ }^{3}$ \\ Jose Luis Sanchez, ${ }^{3}$ and Kishin Sadarangani ${ }^{4}$ \\ ${ }^{1}$ Department of Mathematics, Rzeszów University of Technology, Aleja Powstańców Warszawy 8, 35-959 Rzeszów, Poland \\ ${ }^{2}$ Division of Functional Equations, Zielona Góra University, Ulica Prof. Z. Szafrana 4a, 65-516 Zielona Góra, Poland \\ ${ }^{3}$ Department of Mathematics, Central University of Venezuela, Paseo Los Ilustres, Urbanización Valle Abajo, Apartado Postal 20513, \\ Caracas 1020-A, Capital District, Venezuela \\ ${ }^{4}$ Department of Mathematics, University of Las Palmas de Gran Canaria, Campus de Tafira Baja, \\ 35017 Las Palmas de Gran Canaria, Spain \\ Correspondence should be addressed to Józef Banaś; jbanas@prz.edu.pl
}

Received 11 January 2015; Accepted 11 January 2015

Copyright (C) 2015 Józef Banaś et al. This is an open access article distributed under the Creative Commons Attribution License, which permits unrestricted use, distribution, and reproduction in any medium, provided the original work is properly cited.

Miscellaneous types of function spaces appear very frequently in several mathematical investigations. For example, function spaces create the fundamentals of the study in functional analysis, theory of real functions, theories of differential and integral equations, operator theory, nonlinear analysis, and control theory. Let us also mention that such modern branches of mathematics as numerical analysis and probability theory exploit also methods and tools of the theory of function spaces.

This special issue presents a lot of ideas appearing in the above quoted branches of mathematics. It contains twentytwo papers devoted mainly to the study of function spaces and their various properties. Moreover, this special issue includes also a group of papers discussing some aspects of operator theory in connection with properties of function spaces in which those operators are investigated. Moreover, a part of papers included in this issue is dedicated to the solvability of some functional equations (differential, integral, etc.) and to properties of solutions of those equations.

The first part of the papers, which are devoted to various topics of operator theory in miscellaneous function spaces, contains eight papers. Below we describe briefly those papers. The paper of J. Huang and Y. Liu discusses a molecular characterization of the Hardy space associated with the socalled twisted convolutions. The results of the paper extend several ones obtained by the first author and other authors. An application to the boundedness of local Riesz transforms on the Hardy spaces is also presented. Another paper of the discussed part is authored by S. J. Chang et al. In that paper the analysis of a generalized analytic Feynman integral and a modified generalized analytic functions space associated with the Feynman integral is conducted. Some integration formulas for that integral are established and the applicability to physical circumstances is indicated. J. Dong et al. discuss in their paper the boundedness of singular integrals associated with Schrödinger operators on Hardy type function spaces. The main tool used in the investigations is a molecular characterization of Hardy spaces. The paper of T. Acar et al. describes a new type Stancu operators which create the generalization of Srivastava-Gupta operators. With help of those operators an approximation of functions being integrable on the interval $(0, \infty)$ can be realized. Moreover, the rate of convergence of the approximations in question for functions with derivatives of bounded variation is estimated. $\mathrm{X}$. Feng et al. discuss in their paper a multiplication operator with a special symbol on the weighted Bergman space of the unit ball in $\mathbb{C}^{n}$. A few necessary and sufficient conditions for the compactness of the mentioned multiplication operator are given. In the paper of M. Nowak some general representation theorems for continuous linear operators acting from 
a suitable function space into a Banach space are obtained. Moreover, strongly bounded operators are also studied. The mentioned function space contains vector-valued continuous functions defined on a completely regular Hausdorff space with values in certain Banach space. The paper of J. Xu and $\mathrm{X}$. Yang studies new type of Herz-Morrey-Hardy spaces with variable exponent. Those spaces are characterized in terms of atom. With the help of that characterization a few results on the boundedness of some singular integral operators defined on spaces in question are derived. The other paper included in the discussed group is authored by S. He et al. That paper contains some results concerning the boundedness of some fractional integrals on an infinitesimal generator of an analytic semigroup defined on the Hilbert space of Lebesgue type.

Now, we are going to present the group of six papers dedicated to investigations connected with the theory of function spaces. One paper included in this group is the paper of $\mathrm{H}$. Wang and $\mathrm{Z}$. Wu. The authors deal with the estimates of the $L_{p}$ modulus of continuity of some classes of functions of bounded Waterman-Young variation. The obtained results are applied in obtaining some estimates of Fourier coefficients of functions of the mentioned classes, among others. A. M. Sarsenbi and P. A. Terekhin obtained in their paper general conditions ensuring that a complete biorthogonal conjugate system forms a Riesz basis. Moreover, affine Riesz bases are constructed with the help of the obtained results. The paper of J. Zhou discusses new spaces of Lebesque measurable functions on the unit circle. That space is closely related to a Sobolev space. A few results expressed in terms of Möbius boundedness in a Sobolev space are derived. Moreover, a dyadic characterization of functions of the introduced new space with the aim of dyadic arcs on the unit circle is also presented. In the paper of $\mathrm{X}$. Guo the representation of $g$-frames as linear combination of simpler components ( $g$-orthonormal bases, $g$-Riesz bases, and normalized tight $g$-frames) is considered. Moreover, the dual and pseudodual $g$-frames are investigated and the dual $g$-frames are characterized in a constructive way. Y. Niu and $\mathrm{H}$. Wang study in their paper properties of functions in the class of functions with $p$-bounded Wiener variation for $0<p<1$. The main result asserts that each such function can be represented as the difference of two increasing functions from that class. The paper of Z. Pavić deals with convex functions which satisfy some global convexity properties. The classical ideas associated with Jensen approach to convexity are extended and studied in the paper in question.

Two papers published in this special issue are mainly devoted to operators acting in some function spaces. One paper of that kind authored by O. Mejía et al. deals with a necessary and sufficient condition on a real function $h=h(t)$ such that the composition operator $H$ generated by the function $h$ maps the space of functions with bounded SchrammKorenblum variation into itself and is locally Lipschitzian. Another announced paper of L. Zhou and J. Lu contains a result which creates a generalization of the result of Krues and Zhu concerning the boundedness of an integral operator in the Lebesgue space $L^{p}$.
The fourth group of the papers included in this special issue is formed by six papers devoted thoroughly to some differential and integral equations in various function spaces. One paper written by Z. Dai et al. is dedicated to the Cauchy problem for the three-dimensional incompressible Boussinesq equation. A blow-up criterion for weak solutions of that equation in terms of the pressure is established in a homogeneous Besov space. Another paper by J. Wang et al. investigates a class of singular boundary value problems of a fractional $q$-difference equation. Using a fixed point theorem in partially ordered sets a few results on the existence and uniqueness of solutions of the mentioned equation are established. The paper of Y. Wu et al. shows how to obtain limit cycles for a family of generalized nilpotent systems of differential equations. The results of the paper are well motivated and appropriately illustrated. The paper of $\mathrm{M}$. A. Darwish and B. Rzepka deals with the solvability of a generalized fractional quadratic functional-integral equation of Erdélyi-Kober type in the Banach space of functions being continuous and bounded on the real half-axis. The technique of measures of noncompactness is the main tool used in considerations. T. Zając studies in his paper the existence of nonnegative and monotonic solutions of a nonlinear quadratic Volterra-Stieltjes integral equation. That equation is considered in the classical space consisting of continuous real functions defined on a bounded, closed interval. The main tools used in considerations are the techniques of Stieltjes integrals and measures of noncompactness. The other paper included in the group in question is authored by N. K. Ashirbayev et al. In that paper it is shown that some classes of nonlinear integral equations (integral equations of fractional order, integral equations of Volterra-Wiener-Hopf type, integral equations of Erdélyi-Kober type, and integral equations of Volterra-Chandrasekhar type) can be treated as spacial cases of some nonlinear integral equation of VolterraStieltjes type. Some results concerning Volterra-Stieltjes integral equations in several variables are also discussed.

\section{Acknowledgment}

The guest editors of this special issue would like to express their immense gratitude to the authors who have submitted papers for considerations. We hope that results of the papers included in this special issue will inspire researchers for further study in a lot of branches of mathematical sciences and their applications in describing real world phenomena.

Józef Banaś
Janusz Matkowski
Nelson Merentes
Jose Luis Sanchez
Kishin Sadarangani




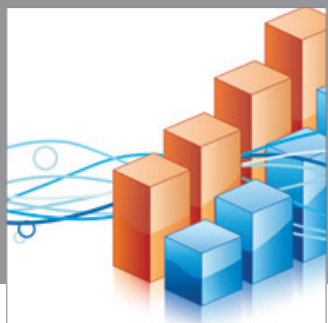

Advances in

Operations Research

mansans

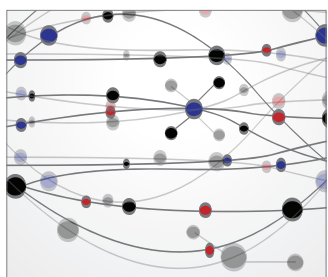

The Scientific World Journal
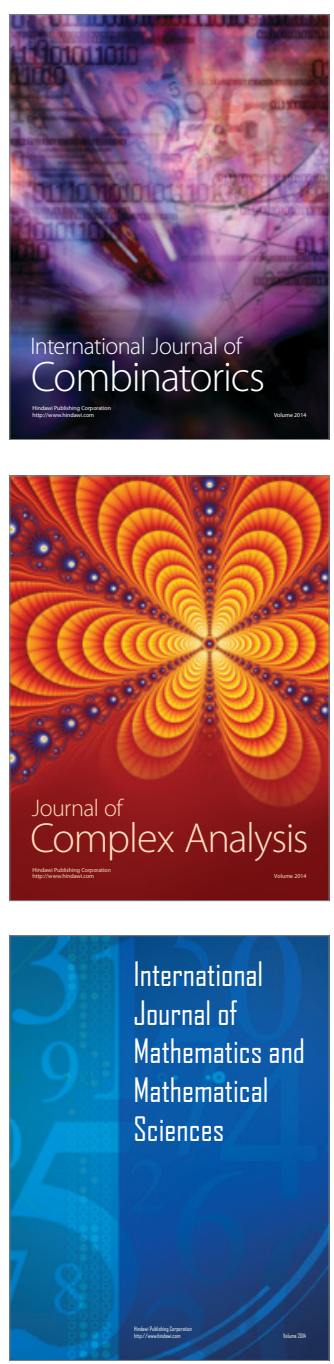
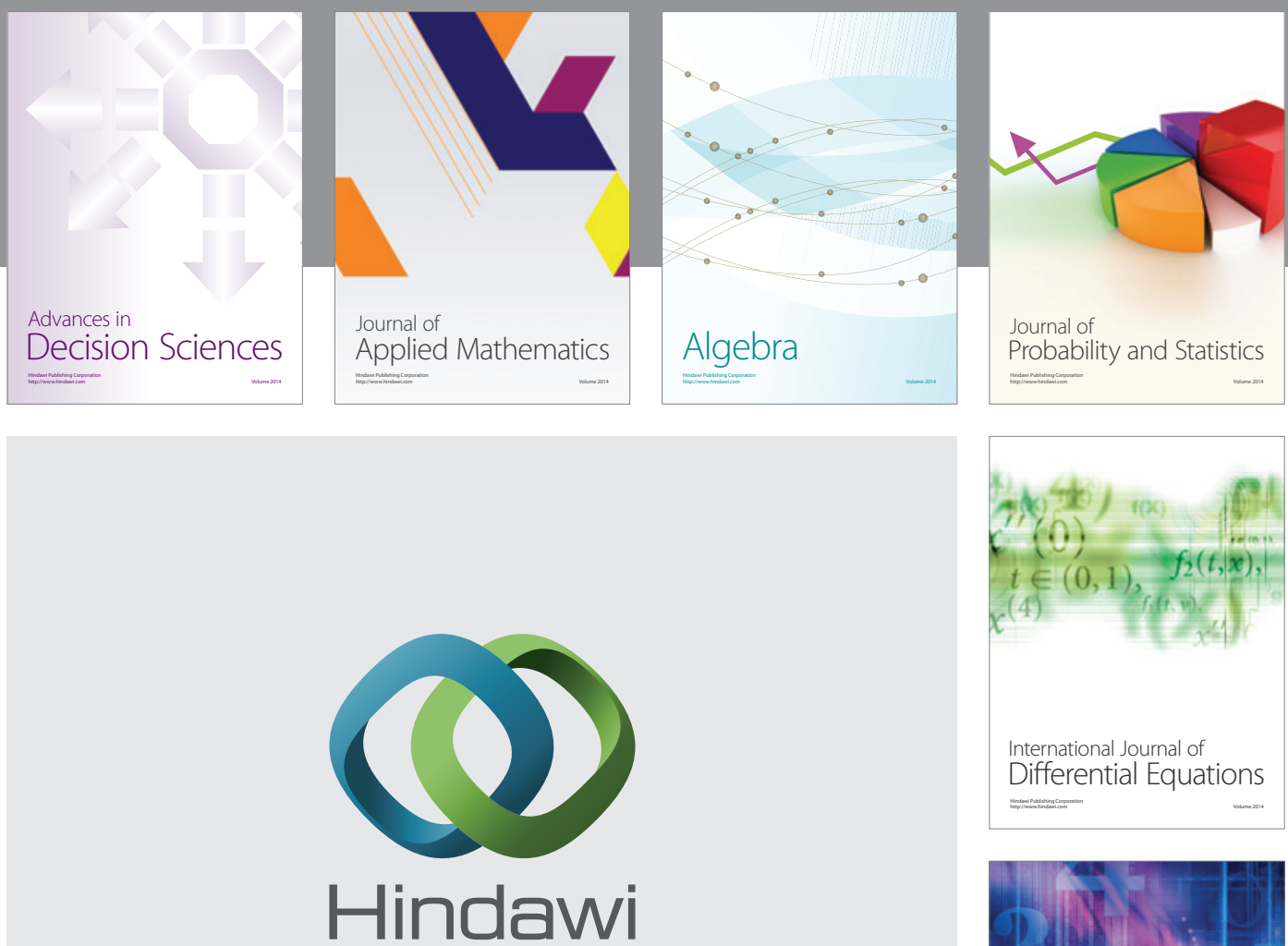

Submit your manuscripts at http://www.hindawi.com
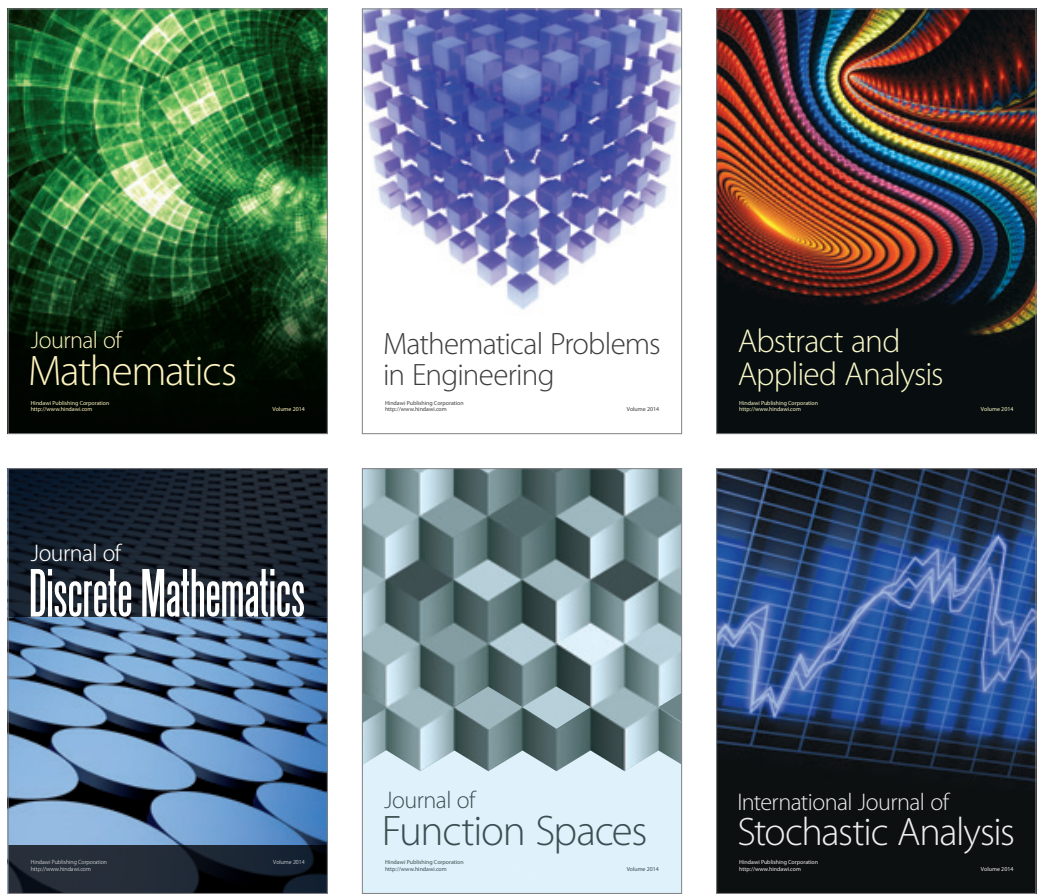

Journal of

Function Spaces

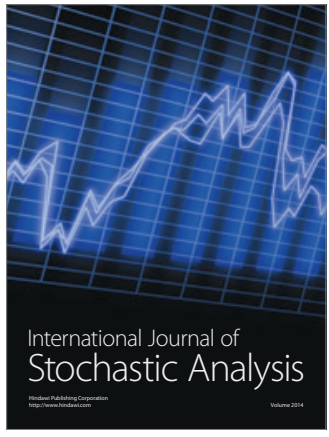

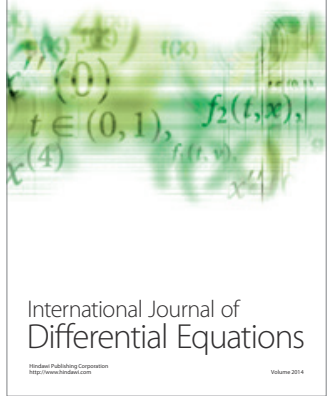
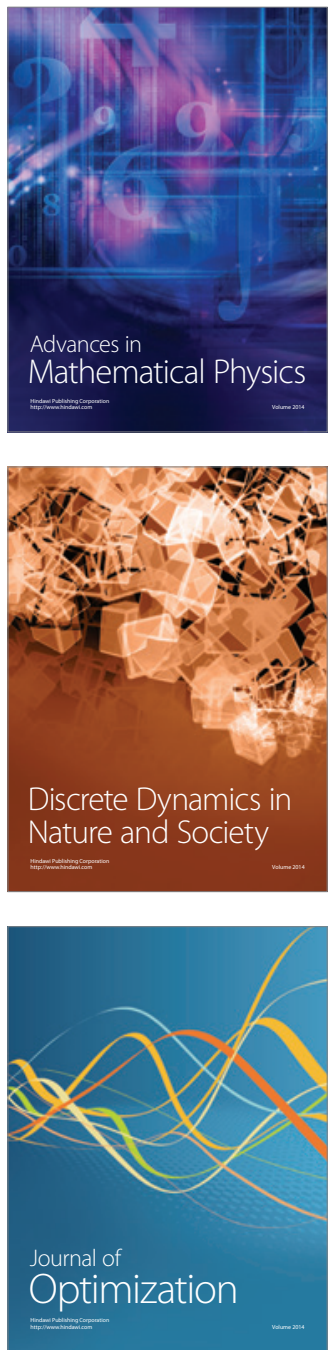